\title{
Review of: "Structural basis for topological regulation of $\mathrm{Tn} 3$ resolvase"
}

\author{
Makkuni Jayaram ${ }^{1}$ \\ 1 University of Texas at Austin
}

Potential competing interests: The author(s) declared that no potential competing interests exist.

This paper by Montano et al. present new biochemical and structural data that form the basis for a revised model with a more complete view of the synaptosome assembled by Tn3 resolvase-a site-specific recombinase of the 'small seine recombinase' family. The present model sheds light on how the recombinase utilizes DNA topology for regulation of its activity. This work advances our current understanding of how a subset of site-specific recombinases senses DNA topology, and imposes a unique 'topological filter' in organizing the partner target sites into a functional recombination synapse. Given the basic biology and synthetic biology interest in site-specific recombinases, the findings will be of general interest to a broad audience.

\section{Specific comments}

1. The structural component of this study involves the crystal forms of Tn3 resolvase-Site III complex in two different space groups. A partial model obtained by molecular replacement was improved by single isomorphous replacement (SIR) and model-derived phases. The placement of amino acid side chains was facilitated by previously solved structures of gd-resolvase. A key feature of the structure is the consistent docking of the $\mathrm{E}$ helix from the right resolvase subunit into the DNA minor groove, although the DNA binding domain (DBD) motifs of the right and left subunits are nearly symmetrical. This asymmetry is likely imposed by the spacer sequence at the center of Site IIII. The docking of the E-helix into the minor groove to widen it and to bend the DNA away from the protein is also seen in the crystal structures of other serine site-specific recombinases, Sin-Site II complex and gd-resolvase-Site I complex. The only weakness of the structural data was the poor density of many of the amino acid side chains. The structure of the closely related gd-resolvase has alleviated this problem.

2. An interesting contrast between the Tn3-resolvase and Sin recombinase structures is in the extent of contacts between the DBDs of two dimers. The contact is extensive for Sin recombinase, but much less for Tn3 resolvase. The findings are consistent with a previous study suggesting that DBD-DBD contacts are not essential for synaptosome formation by Tn3-resolvase. Is there evidence for or against DBD-DBD contacts being critical for synaptosome formation by the Sin-recombinase? Or, are these incidental contacts induced by the different organization of the Sin target sites and the DNA bending caused by IHF? 3. Tn3-resolvase-Site II structure was modeled from the Site III complex by flipping the relative left-to-right 
orientation and including the crystal structure of an AT track to mimic the run of ATs in the additional $9 \mathrm{bp}$ DNA of Site II spacer. The modeling is checked against SAXS data for the fully formed Tn3-resolvase-Site II complexes. Potential discrepancies are ironed out by assuming that the DNA in the isolated Site II complex is less (or less stably) bent than in the synaptosome. Perhaps the statement that the 'SAXS data strongly support the primary features of the Site II complex' should be tempered a bit. The SAXS data are consistent with the model for the Site II complex would be more appropriate.

4. By combining the Site III-complex structure solved in this study, the model for the Site II-complex derived from this structure and SAXS and the previously solved structure for the gd-resolvase-Site I complex, the authors propose a structure of the synaptosome formed by Tn3-resolvase dimers bound to Sites I, II and III of the partner target sites. The connectivity of the bound DNA would constrain Sites II to be in the middle with sites I and III at the base and top of the synaptosome, respectively. The model agrees with the -3 topology (three right handed plectonemic supercoil crossings) of the synaptic structure derived previously from the products of iterated recombination evens without synapse dissociation. The DNase I footprinting of the pre-assembled and glutaraldehyde-crosslinked synaptosome is remarkably similar to published footprinting data for Tn3/gd-resolvase bound to Site I-II-III and to Site II. Enhanced short regions of DNA cleavage specific to the synaptosome can be accommodated by two increased synaptosomespecific DNA bends at locations that are not directly contacted by protein.

5. The validity of the modeled synaptosome structure was tested by a clever set of in vivo recombination assays using Tn3-Bart hybrid resolvases that can be targeted to specific Site I, II or III positions, and whose catalytic domain specificity is determined by a small surface patch of R interface residues form the Tn3 or Bart resolvase. Efficient recombination requires $\mathrm{R}$ interface competence at IR, IIL and both IIIL and IIIR but not IL and IIR. Furthermore, R interface compatibility of subunits at IIIR and IR in trans (that is IIIR of one res site and IR of the partner re site) is critical for efficient recombination. The evidence for interaction between subunits at IIIL and IIL and the requirement of R interface compatibility between them is indirect because R-deficient dimers targeted to site II have a relatively weak effect on recombination. However, when subunits at IR and IIIR are mismatched for R interface in trans, R interface match for subunits at II and IIIL in trans helps recombination. The IR-IIIR and IL-IIIL trans interactions and the dispensability of a functional R interface at IIR and IL are consistent with the proposed model for the synaptosome. It is impressive that the in vivo targeting of resolvase subunits to specific half-sites works well enough to be able to decipher interactions among localized subunits. Given that resolvase is a dimer in solution, unintended targeting due to cooperativity of binding could have been a problem. It is also somewhat surprising that dimerization is apparently unaffected by the Tn3-bart hybrid combinations used for the in vivo assays. One wonders whether or not it is possible to perform the targeting assays in vitro.

6. The apparently delicate architecture of the synaptosome is argued by the authors to be of functional relevance in the biological context of Tn3 resolvase action. Rapid dissociation of the synaptosome after one round of 180 degree rotation by the IL-bound subunits (favored by the limited inter-dimer interactions) would suit the biological role of Tn3 resolvase in processing the cointegrate intermediate formed during 
replicative transposition of Tn3. What about the situation when non-homology of the spacer induces 2 or (2 $x \mathrm{n}$ ) rounds of rotation? One would expect rapid dissociation to produce broken DNA molecules as a result of interrupted recombination. On a related note, is iterated recombination (without synapse dissociation) more frequent for the Sin recombinase, whose synaptosome has a much more compact organization? 7. It would seem that the synaptosome structure can be assembled equally well in a positively supercoiled plasmid substrate as in a negatively supercoiled one. Of course, the plectonemic supercoiled crossings here will be left handed (with + signs). The 180 degree relative rotation of the cleaved recombination complex will be left handed, yielding a +2 catenane as the product. Is there anything about the resolvase structure that would induce a preference for negatively supercoiled DNA (which is the natural situation). For example, is there some feature of the subunit interface that would favor right handed rotation, which can be promoted by negative supercoling?

8. The concluding paragraph of the Discussion with its genome engineering angle would seem a bit exaggerated. What sort of 'further engineering' based on the synaptosome structure is envisaged to utilize Tn3 resolvase to perform targeted insertions, deletions and inversions? I am not sure that the comparison to CRISPR-Cas9, intended to induce the cell to perform homologous DNA repair from a double strand break, is quite valid. The essence of site-specific recombination is DNA rearrangement by precise strand breaks and reunion, leaving no loose ends to be repaired.

\section{Summary}

This work embodies structural analysis in combination with clever biochemical assays to reconstruct the DNA-protein organization of the Tn3-resolvase synaptosome. The assembly differs from that of the Sin recombinase synaptosome but retains the -3 topological filter, which is characteristic of both. It is pleasing to see how the present studies, together with prior biochemical and structural information from related site-specific recombinases, have clarified our perspective of the DNA-protein associations and proteinprotein interactions within a site-specific recombination machine. 